\title{
MRI findings in ruptured ovarian ectopic pregnancy: an unexplored avenue
}

\author{
Annu Singhal ${ }^{1 *}$, Vivek C. Kottiyath ${ }^{2}$, Tej Prakash Gupta ${ }^{3}$, Prachi Arora ${ }^{4}$
}

\author{
${ }^{1}$ Department of Radiodiagnosis, Atal Bihari Vajpayee Institute of Medical Sciences and Dr. Ram Manohar Lohia \\ Hospital, New Delhi, India \\ ${ }^{2}$ Department of Radiodiagnosis, Sky Dianostics, Uttam Nagar, Delhi, India \\ ${ }^{3}$ Department of Radiodiagnosis, Saral Diagnostics, Saraswati Vihar, Delhi, India \\ ${ }^{4}$ Department of Gynaecology and Obstetrics, Bhagwan Mahavir Hospital, Rani Bagh, Delhi, India
}

Received: 21 September 2021

Accepted: 13 October 2021

\author{
*Correspondence: \\ Dr. Annu Singhal, \\ E-mail: annu.singhal@gmail.com
}

Copyright: (C) the author(s), publisher and licensee Medip Academy. This is an open-access article distributed under the terms of the Creative Commons Attribution Non-Commercial License, which permits unrestricted non-commercial use, distribution, and reproduction in any medium, provided the original work is properly cited.

\begin{abstract}
Ovarian ectopic pregnancy (OEP) is a rare form of ectopic pregnancy (EP) and constitutes approximately $0.5-3 \%$ of all ectopic cases. Its presentation mimics the symptoms of tubal ectopic pregnancy, hemorrhagic ovarian cyst/follicle, tuboovarian abscess, urinary tract calculi, appendicitis or ovarian torsion. Occasionally determining the anatomic location of an extra-tubal ectopic pregnancy based on ultrasound imaging and presentation alone can be challenging, particularly when it is adherent to the fallopian tube. Although transvaginal ultrasound (TVS) is the primary modality used in the diagnosis, various forms of OEP and its complications may be incidentally detected and further evaluated on computed tomography (CT) or magnetic resonance imaging (MRI) when an alternative diagnosis is suspected. We reported a case of a second gravid para zero, 25 years old lady, who came with pain in the left lower abdomen. Her urine pregnancy test was positive. TVS showed empty uterine cavity, an extremely tender, heterogenous hyperechoic right adnexal mass, but no obvious gestation sac (GS). A large hematoma was detected adjacent to it in the pouch of Douglas (POD). Keeping a high suspicion of ectopic pregnancy, MRI was performed to evaluate the lesion better which revealed a natural, non-assisted, ruptured right ovarian ectopic pregnancy and was subsequently confirmed at laparotomy and proven on histopathology. Patient underwent left oophorectomy and discharged on 4th day with uneventful follow up.
\end{abstract}

Keywords: OEP, EP, TVS, Magnetic resonance imaging, Gestation sac, POD, Beta-human chorionic gonadotropin

\section{INTRODUCTION}

Multiple risk factors are known that predispose to OEP. They are prior ectopic gestation, genital infections, prior tubal surgery, use of ovulation induction agents and the use of assisted reproductive techniques. ${ }^{1}$ However, it may be due to a random event that is not associated with any of these risk factors as was seen in our case. Early diagnosis of OEP decreases mortality, surgical intervention and enables nonsurgical conservative treatments to be offered.

In the case presented, the diagnosis of a ruptured EP was presumed due to the abnormal serum human chorionic gonadotropin (beta-hCG) levels, equivocal TVS findings due to painful examination, presence of a hyperechoic bulky ovary with no obvious gestational sac, POD hematoma along with clinical symptoms. MRI findings not only prompted us to consider non-tubal ectopic pregnancy as a potential diagnosis, but also revealed the sight of implantation as ovarian and active contrast pooling suggesting rupture. This case presented an example of an OEP that was not conclusively diagnosed until surgery. As far as we know, there were no reports describing the dynamic contrast enhanced MRI findings of ruptured ovarian pregnancy. Hereby, we reported MRI findings in a case of ruptured ovarian pregnancy. 


\section{CASE REPORT}

A 25 year old (gravida 2 para 0) female came to gynaecology emergency with history of amenorrhoea of 5 weeks and pain in the hypogastrium for last one day. Her urine pregnancy test was positive. She had a syncopal attack just before admission. Her past obstetric history involved a first trimester missed abortion 2 months back. The present pregnancy was a spontaneous conception. There was no history of irregular menstrual bleeding or use of any contraceptive device. No history of any medical illness, abdominal surgeries, pelvic inflammatory disease or tuberculosis was noted. There was no significant family history. On general examination, the patient had pallor, tachycardia with a pulse rate of 92 /minute and BP of $100 / 60 \mathrm{mmHg}$. On abdominal examination, there was guarding with mild rebound tenderness in the hypogastrium and right iliac fossa. Per speculum examination revealed a healthy cervix and vagina. Per vaginum examination was very painful and revealed a normal sized anteverted uterus. POD fullness was noted with positive cervical excitement test. On investigation, her haemoglobin $(\mathrm{Hb})$ was $9.8 \mathrm{gm} / \mathrm{dl}$, total leukocyte count was 11,100 /cumm, platelet count was $1.61 \mathrm{lac} / \mathrm{cumm}$ and beta-hCG value was 2,025 IU/1. Rest of the investigations were within normal limits.
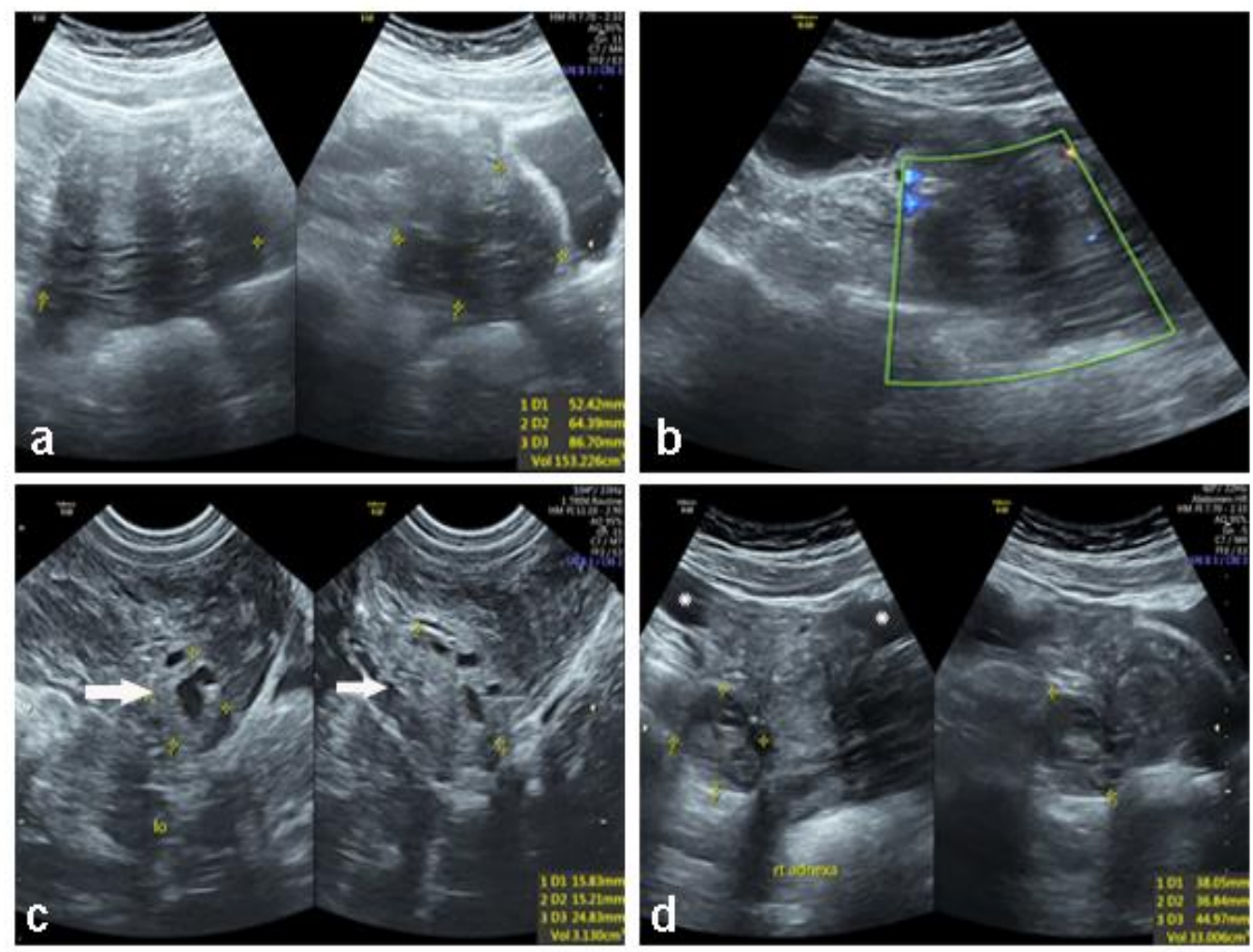

Figure 1: Transvaginal ultrasound (a) a large 153 cc heterogeneously hypoechoic collection with thin septae and low level internal echoes is seen in the POD with (b) no internal/peripheral vascularity; (c) left ovary (lo-arrow) was embedded in its anterosuperior part with small follicles within; (d) bulky right adnexa with a $1.7 \times 1.6 \times 1.4 \mathrm{~cm}$ hyperechoic lesion in posteroinferior part; no obvious ring of fire or central lucency was visualized; free fluid was also seen in the pelvis $(*)$. 

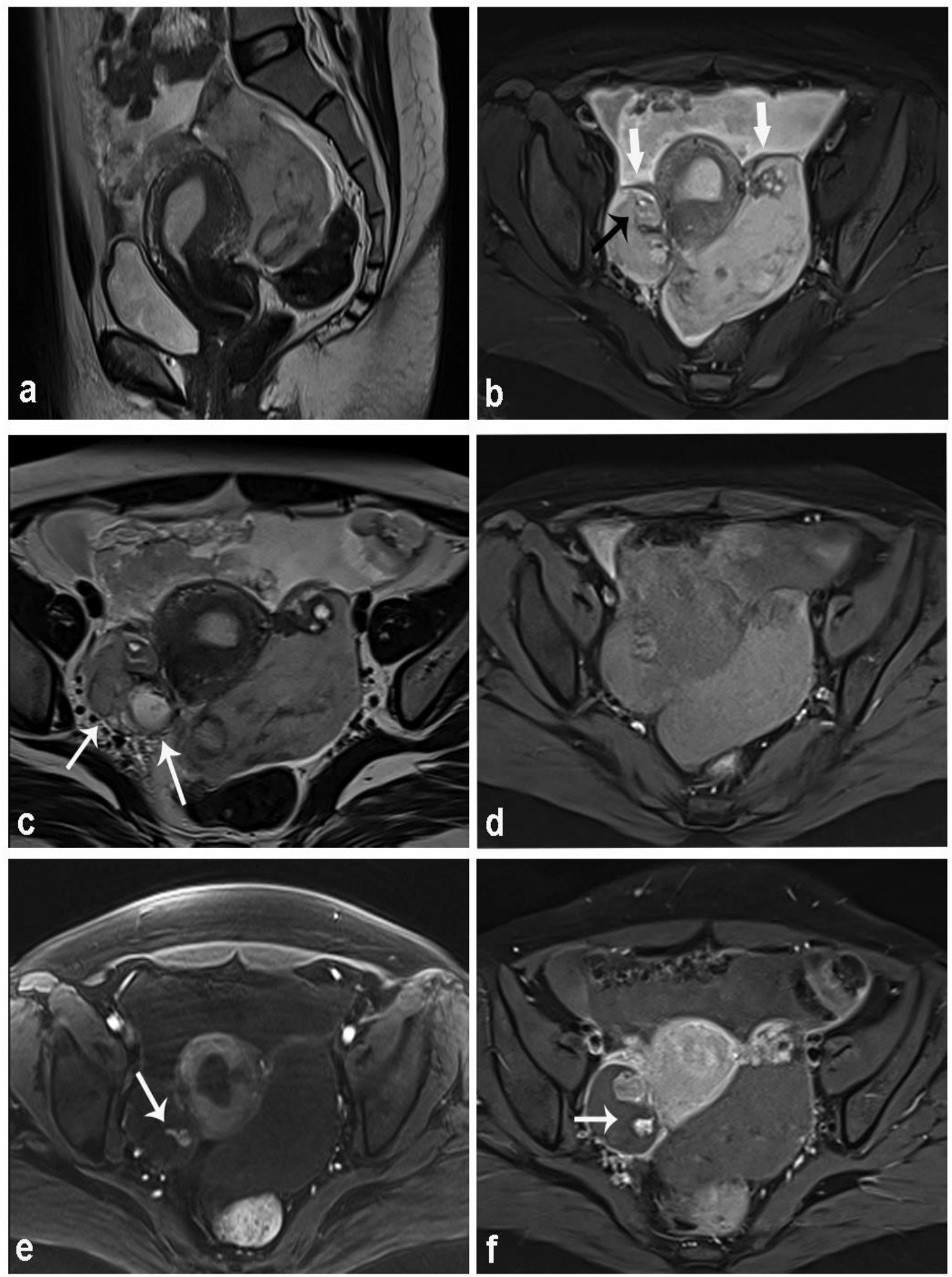

Figure 2: (a) Anteverted, retroflexed uterus is seen with empty cavity; (b) T2 weighted image was showing fluid in the pelvis; a T2 heterogenously hyperintense hematoma was seen in the POD, engulfing the left ovary; both the fallopian tubes were normal (arrows); right ovary was bulky with ovarian tissue in the anterior part (black arrow); a multicystic area (c) was seen in the posteroinferior part (arrows); rest of the ovary appeared hyperintense on $\mathrm{T} 2$ and T1 (d) suggestive of hemorrhage; (e) early arterial phase on dynamic contrast enhanced images showed a small focus of contrast leakage from the cystic part of right ovary (arrow), which enlarged on subsequent venous phase; (f) left ovary and bilateral fallopian tubes showed normal contrast enhancement. 

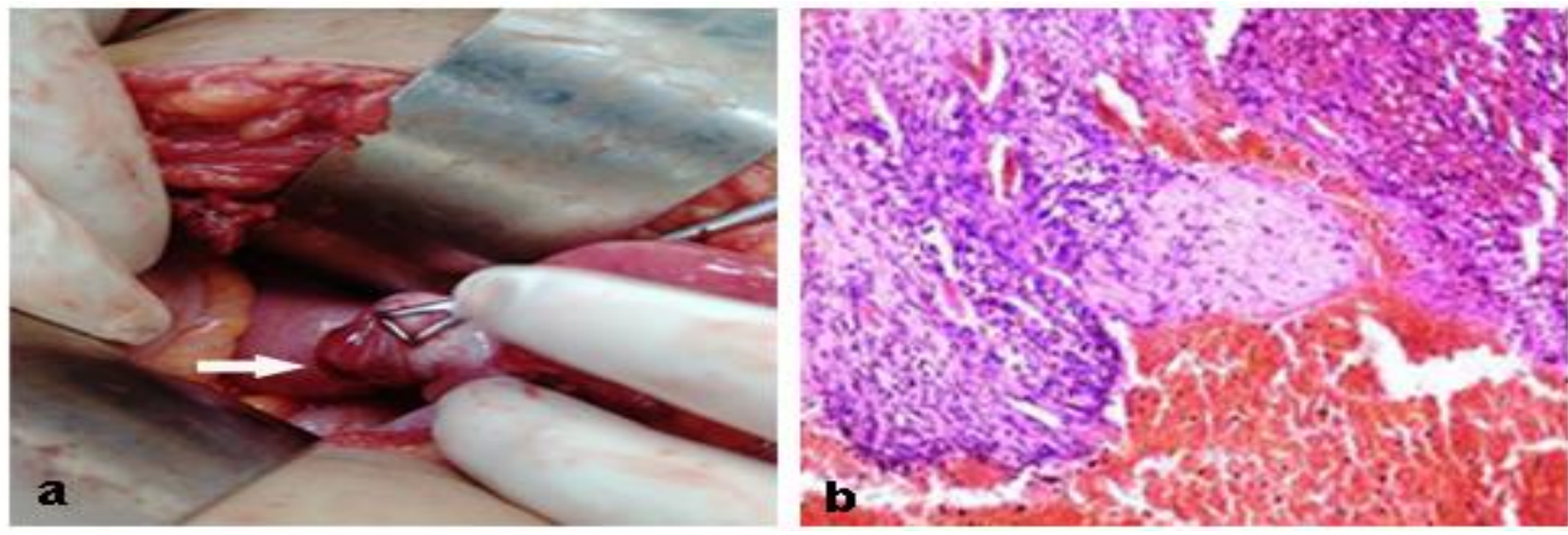

Figure 3: (a) Intraoperative images showed hyperemic right ovary in babcock's forceps with a rent (arrow) suggestive of rupture; (b) histopathology section showing chorionic tissue in the ovarian stroma (H and $\mathrm{E}, \times 100)$.

As a problem solving tool, pelvic MRI was performed in this case. MRI showed free fluid in the pelvis and Morison's pouch. There was a well circumscribed lesion in the POD appearing hyperintense on T2 and T1 weighted images, extending to engulf left ovary in it. Right ovary was bulky, depicting a $19 \mathrm{~mm}$ cystic structure in the posteroinferior part, appearing iso to hyperintense on $\mathrm{T} 2$ and T1 suggestive of hyperacute hemorrhage. Dynamic MR imaging revealed a focus of active, early arterial phase contrast leak suggesting ongoing bleed. Rest of the ovarian parenchyma showed normal enhancement pattern. Right fallopian tube was well seen anterior to the ovary. Left ovary and tube were normal. Uterus was anteverted, retroflexed with empty endometrial cavity. The imaging findings are depicted in Figure 2.

The patient was taken up for emergency laparotomy. Right ovary was bulky and hyperemic with a rent in posteroinferior surface (Figure $3 \mathrm{a}$ ). It was adherent to the tube and attached to the uterus by utero-ovarian ligament. Attempts to completely dissect the right ovary with active bleeding were unsuccessful. It was a small ovary and wedge resection was not possible so right oophorectomy was done. The right fallopian tube was otherwise unremarkable as was the left tube and ovary. Subsequently, histopathological analysis of right ovarian lesion revealed immature placental villi and clots, confirming the presence of ovarian ectopic pregnancy (Figure $3 b$ ). Post-op period was uneventful and the patient was discharged on day 4.

\section{DISCUSSION}

OEP developed following primary or secondary implantation of the fertilized ovum in the ovary. Primary was usually due to ovulatory dysfunction where the ovum was fertilized while still within the follicle, before the follicle being expelled from the ovary. Secondary implantation occured when fertilization takes place within the fallopian tube but the embryo was regurgitated and implanted in the ovarian stroma. This was a result of reverse migration of embryos due to deep deposition of the conceptus into the uterine cavity or the use of an oversized volume of culture fluid during transfer. ${ }^{3,4}$ However, this may be due to a random event that was not associated with any of these risk factors as was seen in our case.

Transvaginal ultrasound was the diagnostic tool for tubal and non-tubal forms of EP. TVS criteria for the presence of OEP were an empty endometrial cavity, an adnexal mass with a GS that was inseparable from ovarian parenchyma, an intraovarian yolk sac and fetal pole, with or without cardiac motion (depending on gestational age), a wide echogenic ring with an internal echolucent area with a ring of fire sign on Doppler within the ovarian cortex, separate from corpus luteum or follicles. ${ }^{2}$ A true ovarian pregnancy showed a negative sliding organ sign, that cannot be separated from the ovarian tissue by applying gentle pressure by endovaginal probe from within or with manual compression on the patient's abdomen by hand. ${ }^{5}$ However, there was some overlap as a tubal pregnancy can also became adherent to the ovary. Other findings can be collection around the ovary, absence of dilated fallopian tube and hemoperitoneum in case of rupture. Confident diagnosis of OEP, however, was based on the positive identification of an extra-tubal mass with GS rather than the absence of an intrauterine GS. Differentials here can be tubal ectopic or a corpus luteal cyst (CLC). A tubal EP ring was much thinner by comparison. The echogenicity of the OEP was more as compared to a CLC. However, we cannot reliably distinguish these two using color Doppler. They both can display the ring of fire sign, as both the ectopic ring and the walls of a corpus luteum can have significant vascularity. Studies have shown that there was no significant difference between the peak systolic velocities (PSV) of an EP compared to that of a corpus luteum. ${ }^{6}$

It may be difficult to determine by TVS alone whether an empty uterus indicated early pregnancy, missed abortion 
or unidentified EP. A completely normal pelvic TVS scan may be present in $15-20 \%$ of patients with ectopic pregnancy. Hence, we must do follow up and clinical correlation with serum HCG values. In such cases, on transabdominal ultrasonography (TAS) and with the patient's beta-hCG level greater than $6,500 \mathrm{mIU}$ per $\mathrm{ml}$ $(6,500$ IU per 1$)$ or on TVS and with the patient's beta-hCG level 1,500 mIU per ml (1,500 IU per l) or greater, a high suspicion of EP should be reported. ${ }^{7}$ In a normal intrauterine pregnancy, these levels increase by at least 53 percent every two days, peaking at a level greater than $100,000 \mathrm{mIU}$ per $\mathrm{ml}(100,000 \mathrm{IU}$ per 1$)$ as compared to in $\mathrm{EP}$, when the rise was much slower and hardly reaches this level. ${ }^{8}$

MRI was primarily performed as a problem solving tool when the information obtained with TVS was inadequate, the results of the MRI may alter patient care and the acuteness of the patient's condition precludes waiting until the pregnancy was completed. ${ }^{9}$ Specific unsurity in OEP diagnosis may arise if there was presence of tubal hematoma or hemoperitoneum, fallopian tube was not separately seen from the adnexal mass, in case of probe tenderness or unstable condition or extrauterine GS was mimicking corpus luteum cyst. In all such cases, MRI can also be useful tool for accurately localizing the implantation site. ${ }^{10}$

Shingo described a GS structure, which exhibited a heterogeneous high intensity intermingled with punctate foci of distinct low intensity on T2-weighted images, incarcerated to the posterior surface of the ovary. The mass formed a beak sign in the ovary, in the absence of a dilated fallopian tube. T1-weighted images revealed foci of high intensity in the mass, corresponding to the low intensity on T2-weighted images, suggesting hemorrhage. ${ }^{10}$ In our case, site of implantation in the ovary was only visualized by MRI. Both the fallopian tubes and left ovary were well visualized separately. The hematoma in the pelvis was better defined with visualization of an active bleeding focus from the ruptured ectopic site.

Even with direct visualization during surgery, an ovarian pregnancy can still be mistaken for a haemorrhagic cyst. Histopathologic confirmation was required to confirm the diagnosis. When determining the management of OEP, careful consideration of the patient's desire to have more children should be given.

\section{CONCLUSION}

The incidence of ovarian pregnancy is increasing with in vitro fertilization rate on its hike and hence one needs to have a high index of suspicion. This may prevent potential rupture and thus a surgical emergency. Various types of ectopic pregnancy have characteristic imaging features. We must always consider the possibility of ovarian ectopic pregnancy in the setting of hemoperitoneum or an adnexal mass with normal tubes, in a woman of child-bearing age with positive urine pregnancy test, even in the absence of risk factors. TVS is the imaging modality of choice; however familiarity with the typical MRI appearances can facilitate conceptus localization, prompt and accurate diagnosis and treatment. Judicious use of MRI in an appropriate clinical setting can thus prevent mishaps and enable better management of such conditions.

\section{Funding: No funding sources \\ Conflict of interest: None declared \\ Ethical approval: Not required}

\section{REFERENCES}

1. Tehrani HG, Hamoush Z, Ghasemi M, Hashemi L. Ovarian ectopic pregnancy: a rare case. Iran J Reprod Med. 2014;12(4):281-4.

2. Choi HJ, Im KS, Jung HJ, Lim KT, Mok JE, Kwon YS. Clinical analysis of ovarian pregnancy: a report of 49 cases. Eur J Obstet Gynecol Reprod Biol. 2011;158(1):87-9.

3. Rathore R, Bansal R, Sharma R, Jindal R, Nargotra N. Ovarian ectopic pregnancy-a report of 2 cases and review of literature. Asian Pac J Health Sci. 2017;4(2):110-4.

4. Singh N, Sarmalkar M, Mehendale M, Nayak A. Ruptured primary ovarian ectopic pregnancy: a case series. Int J Reprod Contracept Obstet Gynaecol. 2016;5(10):3641-4.

5. Stein MW, Ricci ZJ, Novak L, Roberts JH, Koenigsberg M. Sonographic comparison of the tubal ring of ectopic pregnancy with the corpus luteum. J Ultrasound Med. 2004;23(1):57-62.

6. Tamai K, Koyama T, Togashi K. MR features of ectopic pregnancy. Eur Radiol. 2007;17(12):3236-46.

7. Borrelli PT, Butler SA, Docherty SM, Staite EM, Borrelli AL, Iles RK. Human chorionic gonadotropin isoforms in the diagnosis of ectopic pregnancy. Clin Chem. 2003;49(12):2045-9.

8. Roy J, Babu AS. Ovarian pregnancy: two case reports. Australas Med J. 2013;6(8):406-10.

9. Takahashi A, Takahama J, Marugami N. Ectopic pregnancy: MRI findings and clinical utility. Abdomin Imaging. 2013;38(4):844-50.

10. Io S, Hasegawa M, Koyama T. A case of ovarian pregnancy diagnosed by MRI. Case Rep Obstetr Gynecol. 2015;2015:14303.

Cite this article as: Singhal A, Kottiyath VC, Gupta TP, Arora P. MRI findings in ruptured ovarian ectopic pregnancy: an unexplored avenue. Int J Reprod Contracept Obstet Gynecol 2021;10:4323-7. 